\title{
AVALIAÇÃO DO USO DOS ANTIOXIDANTES COMERCIAIS BHT E IONOL NA ESTABILIDADE OXIDATIVA DE SEBO BOVINO
}

\author{
Maurício Nunes Kleinberg ${ }^{1}$, Maria Alexsandra De Sousa Rios ${ }^{2}$, Francisco Murilo Tavares \\ De Luna ${ }^{2}$, Marcelo Monteiro Valente Parente ${ }^{3}$, Hugo Leonardo De Brito Buarque ${ }^{4}$ \\ ${ }^{1}$ Instituto Federal de Educação, Ciência e Tecnologia do Ceará (IFCE), Campus de Maracanaú \\ ${ }^{2}$ Universidade Federal do Ceará (UFC) \\ ${ }^{3}$ Instituto Federal de Educação, Ciência e Tecnologia do Ceará (IFCE), Campus de Caucaia \\ ${ }^{4}$ Instituto Federal de Educação, Ciência e Tecnologia do Ceará (IFCE), Campus de Fortaleza \\ $<$ mauricionkleinberg@gmail.com>, \\ $<$ alexsandrarios@ufc.br>,<murilo@gpsa.ufc.br>, \\ $<$ marceloparente2@gmail.com>,<hbuarque@ifce.edu.br>
}

DOI: 10.21439/conexoes.v10i5.1163

\begin{abstract}
Resumo. O sebo bovino é a segunda maior fonte lipídica para produção de biodiesel no Brasil. Contudo, problemas relacionados à rancificação hidrolítica e oxidativa do sebo, os quais ocorrem em geral na armazenagem deste insumo, podem comprometer a produção e a qualidade do biodiesel formado. Este trabalho experimental buscou avaliar a influência dos antioxidantes comerciais butilhidroxitolueno e IONOL na melhoria da estabilidade oxidativa de sebo bovino. Assim, amostras de sebo bovino, previamente caracterizadas, tiveram seus períodos de indução determinados por método Rancimat, antes e após a adição de $1000 \mathrm{mg} / \mathrm{kg}$ ou $5000 \mathrm{mg} / \mathrm{kg}$ de cada um dos antioxidantes testados. O período de indução do sebo, inicialmente de 10 horas, aumentou com o aumento da dosagem de antioxidante. A melhoria na estabilidade oxidativa do sebo bovino foi maior com a adição de $5000 \mathrm{mg} / \mathrm{kg}$ de IONOL $(44,79$ horas de período de indução). Os antioxidantes comerciais butilhidroxitolueno e IONOL melhoraram a estabilidade oxidativa do sebo bovino.
\end{abstract}

Palavras-chave: Sebo bovino. Biodiesel. Oxidação. Antioxidantes. Rancimat.

\begin{abstract}
The beef tallow is the second largest lipid source for biodiesel production in Brazil. However, problems related to hydrolytic and oxidative rancidity of beef tallow, which usually occur in the storage step of this raw material, may compromise the production and the quality of the biodiesel. This experimental work aimed to evaluate the influence of the commercial antioxidants butylhydroxytoluene and IONOL in improving the oxidation stability of beef tallow. Samples of beef tallow were previously characterized and then had their induction periods determined by the Rancimat method before and after the addition of $1000 \mathrm{mg} / \mathrm{kg}$ or $5000 \mathrm{mg} / \mathrm{kg}$ of each of the antioxidants that were tested. Beef tallow's induction period was originally of 10 hours and increased with increasing antioxidant dosage. The greatest improvement in oxidation stability occurred with the addition of $5000 \mathrm{mg} / \mathrm{kg}$ of IONOL (induction period at 44.79 hours). The commercial antioxidants butylhydroxytoluene and IONOL improved the oxidation stability of beef tallow.
\end{abstract}

Keywords: Beef tallow. Biodiesel. Oxidation. Antioxidants. Rancimat. 


\section{INTRODUÇÃO}

Biodiesel é um combustível biodegradável formado a partir de uma reação química, estimulada por um catalisador, entre fontes renováveis, como gorduras animais e óleos vegetais, e um álcool de cadeia curta, usualmente o etanol ou o metanol (BRASIL, 2004).

Em 2015, o Brasil foi o segundo maior consumidor mundial de biodiesel (3,9 milhões de $\left.\mathrm{m}^{3}\right)$, descontando-se a exportação de aproximadamente 12 mil $\mathrm{m}^{3}$, tendo ficado atrás somente dos Estados Unidos (5,6 milhões de $\mathrm{m}^{3}$ ) (BRASIL, 2016a).

De janeiro a julho de 2016, a produção de biodiesel no Brasil atingiu $1.887 \mathrm{mil} \mathrm{m}^{3}$, um decréscimo de 2,3\% em relação ao mesmo período de 2015 , no qual foram produzidos $1.931 \mathrm{mil} \mathrm{m}^{3}$ de biodiesel (BRASIL, 2016a).

Em 2005, foi criado o Programa Nacional de Produção e Uso de Biodiesel (PNPB) com o objetivo de incentivar de forma sustentável o uso e a produção de biodiesel no Brasil, a partir de diferentes matériasprimas oleaginosas em regiões diversas, com foco em promover a inclusão social (MARTINS et al., 2011).

O uso de óleos vegetais, a partir da mamona e palma, como principais insumos da produção de biodiesel foram o foco do PNPB, onde o regime de agricultura familiar foi uma prioridade para garantir a inclusão social. No entanto, o Governo Federal vem tendo dificuldade em atingir as metas deste programa. Enquanto tais dificuldades do Governo Federal não se resolvem, as indústrias de biodiesel vêm consumindo matérias-primas amplamente industrializadas e comercializadas no território brasileiro. Entre as matérias-primas, o sebo bovino tem se destacado desde o início do Programa Nacional de Produção e Uso de Biodiesel como a segunda opção mais utilizada na produção de biodiesel. Em maio de 2016, a matéria-prima que teve maior participação na produção de biodiesel no Brasil foi o óleo de soja, com $80,67 \%$, seguida pela gordura bovina, com $15,29 \%$ e outras matérias-primas, com $4,04 \%$ (BRASIL, 2016b).

Na região Nordeste do Brasil, a gordura bovina ocupa o percentual de $31,21 \%$ entre as matérias-primas utilizadas para produção de biodiesel. $\mathrm{O}$ sebo bovino tornou-se uma alternativa vantajosa devido ao seu custo inferior em relação ao óleo de soja. Além disso, o biodiesel de sebo bovino é mais estável e tem maior cetanagem que o biodiesel de óleo de soja (BRASIL, 2016b; CASTANHEIRA et al., 2014; ORIVES et al., 2014; RINCÓN; JARAMILLO; CARDONA, 2014).

$\mathrm{O}$ sebo bovino é acondicionado nas indústrias à temperatura de aproximadamente $60{ }^{\circ} \mathrm{C}$, visto que abaixo desta temperatura, esta matéria-prima encontra-se na fase sólida, dificultando seu manuseio no processo de produção no qual o mesmo esteja inserido.

O grande problema do sebo bovino como matériaprima são os elevados índices de acidez encontrados, consequência do processo de rancificação hidrolítica e oxidativa, que é significativo na presença de valores elevados de umidade e temperatura, normalmente encontrados no seu processo de armazenagem. Estes índices elevados comprometem o rendimento da produção do biodiesel, principalmente pelo consumo elevado dos catalisadores básicos (geralmente usados) e pela saponificação dos ácidos graxos livres (RINCÓN; JARAMILLO; CARDONA, 2014).

Sabe-se que na reação de transesterificação via catálise básica, a acidez da matéria-prima oleaginosa favorece a formação de reações paralelas indesejáveis, tais como a formação de sabão, que, por ser emulsificante, dificulta consideravelmente o processo de separação da glicerina da fase éster (biodiesel).

Segundo informações fornecidas pela PBIO unidade Quixadá, o sebo bovino ao ser processado na usina de biodiesel, apresentava um acréscimo de 3 a $4 \%$ de acidez na matéria-prima. Como a PBIO, como todas as outras usinas de biodiesel, só processa matériaprima oleaginosa com acidez abaixo de $1 \%$, a PBIO separa o excesso de ácidos contidos no sebo por destilação a vácuo (processo físico), ou por neutralização com lixívia (processo químico). Nos dois processos, são gerados como resíduos o ácido graxo livre e a borra, respectivamente ${ }^{1}$.

O valor aproximado do sebo bovino no Brasil foi de R\$ 2.500,00 por tonelada em 2016, no acumulado até julho (BRASIL, 2016a). Por outro lado, o ácido graxo e a borra são negociados para venda com valores por volta de $\mathrm{R} \$ 800,00$ por tonelada ${ }^{1}$. A capacidade de produção da usina de biodiesel da Petrobras Biocombustível em Quixadá é de 301,71 $\mathrm{m}^{3} /$ dia de biodiesel. Para esta produção são necessárias aproximadamente $270 \mathrm{t} / \mathrm{dia}$ de matéria-prima. Considerando para a usina o mesmo percentual de uso da gordura bovina como matéria-prima na produção de biodiesel que o da região Nordeste de 31,21\%, então seriam necessárias na usina 84 t/dia de sebo bovino (BRASIL, 2016b). Considerando que este sebo apresente aproximadamente $4 \%$ de acidez, conforme valores apresentados anteriormente, isto significa uma perda de matéria-prima por volta de 3,3 t/dia,

\footnotetext{
1 Informação fornecida pelo sr. Antônio Almeida, engenheiro de processamento da usina de biodiesel da Petrobras Biocombustível em Quixadá.
} 
considerando que toda a acidez é retirada de sua composição e comercializada.

Com os valores apresentados anteriormente da compra do sebo bovino e da venda do ácido graxo, esta empresa estará tendo uma perda de aproximadamente R\$ 5.610,00 por dia. Considerando uma produção de 350 dias por ano, a perda anual desta empresa fica por volta de R\$1.963.500,00.

Deste modo, é estratégico estudar a inserção de um antioxidante eficaz e dosagens apropriadas que atuem na estabilização oxidativa do sebo bovino, permitindo, assim, diminuir as perdas ocasionadas pela sua degradação na etapa de armazenamento. O presente trabalho avaliou a influência do uso de dois antioxidantes comerciais, o butilhidroxitolueno (BHT) e o IONOL na estabilidade oxidativa de amostras de sebo bovino por meio de ensaios de período de indução (Método Rancimat).

\section{MATERIAIS E MÉTODOS}

O material sebáceo bovino foi obtido no Frigorífico Aguiar localizado no Quarto Anel Viário na cidade de Maracanaú, Brasil. O sebo bovino utilizado nos experimentos foi extraído a partir do material sebáceo bovino por meio de aquecimento em temperatura de até $120^{\circ} \mathrm{C}$. Com o aquecimento, o sebo bovino sofreu fusão, tornando-se líquido. Foi feita a separação do sebo líquido de demais materiais sebáceos por meio de filtração. O sebo foi armazenado em freezer em temperatura inferior a $-4{ }^{\circ} \mathrm{C}$ até o momento de ser utilizado nos experimentos.

Uma quantidade adequada do sebo bovino refrigerado foi inicialmente aquecida a uma temperatura de $70{ }^{\circ} \mathrm{C}$, aproximadamente, e dividida em alíquotas para a posterior dosagem dos dois antioxidantes avaliados. Então, cinco amostras de sebo bovino foram preparadas para uso nos ensaios de estabilidade oxidativa, que foram as seguintes: sebo bovino sem antioxidante; dosado com BHT nos teores de 1000 $\mathrm{mg} / \mathrm{kg}$ e de $5000 \mathrm{mg} / \mathrm{kg}$; e dosado com IONOL nos teores de $1000 \mathrm{mg} / \mathrm{kg}$ e de $5000 \mathrm{mg} / \mathrm{kg}$. Todos os ensaios de período de indução foram executados em duplicata ou em número maior de repetições.

Os antioxidantes comerciais usados nos experimentos foram: o butilhidroxitolueno $\geq 99 \%$, obtido da Dinâmica Química Contemporânea Ltda., Brasil, e o IONOL (mistura composta por $86 \%$ de 2,6-di-tercbutilfenol e $14 \%$ por 2,4,6-tri-terc-butilfenol), obtido da Sigma-Aldrich, Brasil (SANTOS, 2013; SOUZA et al., 2011). As estruturas moleculares dos dois antioxidantes utilizados são apresentadas na Figura 1.
Todos os demais reagentes, utilizados na caracterização do sebo, eram de grau analítico ou superior.

\section{Figura 1 - Estruturas moleculares dos antioxidantes comerciais avaliados.}

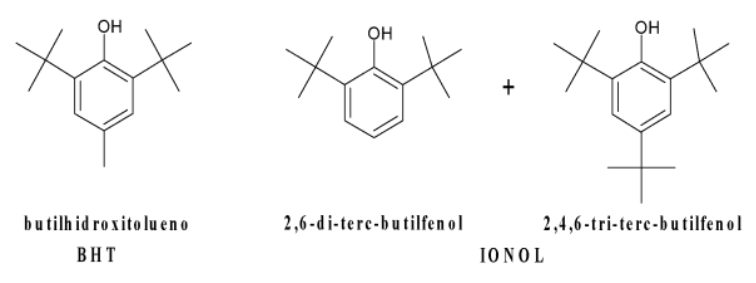

Previamente aos ensaios de estabilidade oxidativa realizados, o sebo bovino sem antioxidante foi caracterizado quanto às seguintes propriedades físicoquímicas: índice de acidez total (método titulométrico, norma AOCS 3d-63 (AMERICAN OIL CHEMISTS' SOCIETY, 2013), com as modificações sugeridas por Moretto e Fett (MORETTO; FETT, 1998)); índice de peróxido (método titulométrico, norma AOCS 8-53 (AMERICAN OIL CHEMISTS' SOCIETY, 1990)); índice de iodo (método titulométrico, norma NBR 9.231 (ASSOCIAÇÃO BRASILEIRA DE NORMAS TÉCNICAS, 2012)); e índice de saponificação (método titulométrico, norma NBR 10.448 (ASSOCIAÇÃO BRASILEIRA DE NORMAS TÉCNICAS, 2012)).

A estabilidade oxidativa das amostras de sebo bovino sem antioxidante e com a adição dos antioxidantes foi avaliada usando-se um equipamento Rancimat (modelo 873 Biodiesel Rancimat ${ }^{\circledR}$, marca Metrohm, Suíca) segundo a norma EN 14112 (BSI BRITISH STANDARDS, 2003). Para o preparo das amostras para os testes no Rancimat, as alíquotas do sebo foram aquecidas até a temperatura aproximada de $70^{\circ} \mathrm{C}$ e foram adicionados os antioxidantes nas concentrações de $1000 \mathrm{mg} / \mathrm{kg}$ e $5000 \mathrm{mg} / \mathrm{kg}$. Inicialmente, água deionizada é colocada em recipientes, os quais são colocados no Rancimat para medir a condutividade da água deionizada. Para poder utilizar a água deionizada, a sua condutividade deve medir inicialmente o valor máximo de $5 \mu \mathrm{S} / \mathrm{cm}$. Essencialmente, o processo de oxidação acontece dentro de um tubo de reação aquecido à temperatura de $110^{\circ} \mathrm{C}$ (com 3 gramas da amostra de sebo bovino) com injeção de ar à $10 \mathrm{~L} / \mathrm{h}$. Os produtos voláteis da oxidação formados durante o experimento de oxidação são coletados em um recipiente contendo $50 \mathrm{~mL}$ de 
água deionizada, e a condutividade da água deionizada é monitorada continuamente. Uma mudança brusca na condutividade elétrica desta água é observada no período de indução, assim indicando o início da propagação de um processo oxidativo. Após este ponto, são observados um rápido crescimento na taxa de oxidação, índice de peróxido e absorção de oxigênio (ARAÚJO et al., 2011). Os ensaios finalizaram quando a condutividade elétrica da solução aquosa chegou a $200 \mu \mathrm{S} / \mathrm{cm}$.

\section{RESULTADOS E DISCUSSÃO}

Os resultados obtidos da caracterização físico-química do sebo bovino utilizado nos ensaios de estabilidade oxidativa são apresentados na Tabela 1 .

Tabela 1 - Resultados de análises físico-químicas do sebo bovino sem antioxidante antes da oxidação.

\begin{tabular}{l|c|c|c|}
\hline \multicolumn{1}{c|}{ Caracteristica } & Resultado & Unidade & Referência \\
\hline Índice de Acidez Total & 1,01 & $\mathrm{mg} \mathrm{KOH} / \mathrm{g}$ & 3,30 \\
\hline Índice de Saponificação & 197,56 & $\mathrm{mg} \mathrm{KOH} / \mathrm{g}$ & 246,10 \\
\hline Índice de Iodo & 26 & $\mathrm{~g} \mathrm{I}_{2} / 100 \mathrm{~g}$ & $33-47$ \\
\hline Índice de Peróxido & 9,60 & $\mathrm{meq} / \mathrm{kg}$ & 5,00 \\
\hline
\end{tabular}

Fonte: Próprio autor. IFCE, 2016.

Nota: A coluna de referência contém dados da literatura para fins comparativos. Fonte: (FARIAS et al., 2012; PINTO, 2014).

Pode ser observado que o sebo bovino utilizado apresenta características típicas de gorduras animais, com destaque para o índice de iodo que apresentou resultado menor que o limite inferior de referência, indicando que o sebo obtido possui um teor de insaturados menor que o usual para este tipo de gordura.

Seguindo a metodologia já descrita, curvas de indução oxidativa foram obtidas a partir dos ensaios de estabilidade oxidativa para todas as amostras preparadas. Algumas dessas curvas cinéticas obtidas para o sebo bovino dosado com antioxidantes, os valores médios de período de indução e seus respectivos desvios padrões estão mostrados nos diagramas da Figura 2. Uma curva do sebo sem antioxidante também é plotada nesses diagramas.
Figura 2 - Curvas cinéticas de indução oxidativa obtidas em alguns dos ensaios com amostras do sebo bovino dosadas com os antioxidantes avaliados (BHT e IONOL) nas dosagens de 1000 $\mathrm{mg} / \mathrm{kg}$ e $5000 \mathrm{mg} / \mathrm{kg}$.

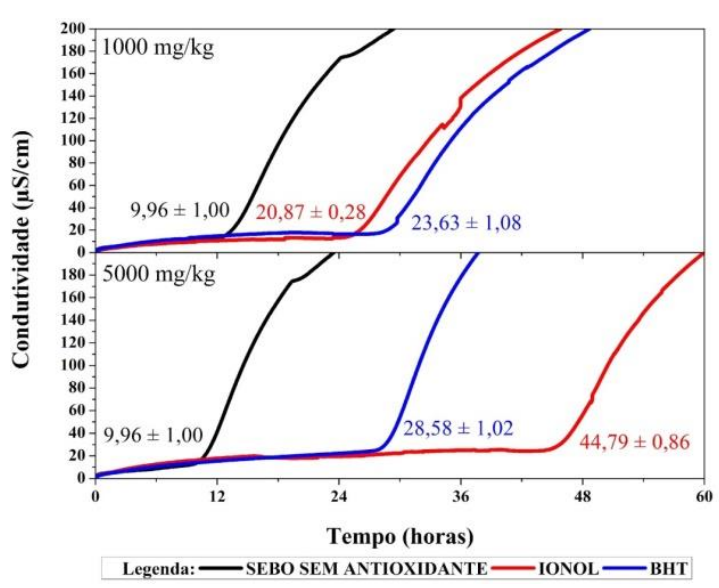

Fonte: Próprio autor. IFCE, 2016.

Os valores médios e os respectivos desvios padrões de período de indução, calculados a partir das curvas de indução determinadas para o sebo bovino sem antioxidante e para o sebo bovino dosado com os antioxidantes nos teores de $1000 \mathrm{mg} / \mathrm{kg}$ e $5000 \mathrm{mg} / \mathrm{kg}$ foram os seguintes: sebo bovino sem antioxidante $(9,96 \pm 1,00)$; dosado com $1000 \mathrm{mg} / \mathrm{kg}$ de BHT $(23,63 \pm 1,08)$; dosado com $1000 \mathrm{mg} / \mathrm{kg}$ de IONOL $(20,87 \pm 0,28)$; dosado com $5000 \mathrm{mg} / \mathrm{kg}$ de BHT $(28,58 \pm 1,02)$; e dosado com $5000 \mathrm{mg} / \mathrm{kg}$ de IONOL $(44,79 \pm 0,86)$.

A adição de BHT e de IONOL na menor dosagem aplicada $(1000 \mathrm{mg} / \mathrm{kg})$ duplica o período de indução do sebo bovino. No entanto, o período de indução do sebo bovino dosado com o BHT no teor de 1000 $\mathrm{mg} / \mathrm{kg}$ é apenas um pouco maior do que o período de indução do sebo bovino dosado com o IONOL no teor de $1000 \mathrm{mg} / \mathrm{kg}$.

$\mathrm{O}$ aumento da dosagem de antioxidante nas amostras de $1000 \mathrm{mg} / \mathrm{kg}$ para $5000 \mathrm{mg} / \mathrm{kg}$ provoca um aumento da estabilidade à oxidação da gordura animal avaliada. A adição de $5000 \mathrm{mg} / \mathrm{kg}$ do IONOL foi a que apresentou o maior período de indução, como também foi a que apresentou o maior aumento do período de indução em relação à dosagem de $1000 \mathrm{mg} / \mathrm{kg}$. Vale destacar que a adição do IONOL na maior dosagem testada superou em mais do que 4 vezes a estabilidade 
oxidativa (período de indução) do sebo bovino não dosado.

Em outro estudo realizado pelo grupo de pesquisa, o antioxidante TBHQ também foi avaliado nessa faixa de dosagem (1000 mg/kg e $5000 \mathrm{mg} / \mathrm{kg}$ ), onde o mesmo apresentou maior período de indução comparado ao BHT (ALVES et al., 2015). Vale destacar que para o sebo bovino dosado com 5000 $\mathrm{mg} / \mathrm{kg}$ de TBHQ, o período de indução superou em mais de doze vezes o período de indução do sebo bovino sem antioxidante. Também, o aumento da dosagem de $1000 \mathrm{mg} / \mathrm{kg}$ para $5000 \mathrm{mg} / \mathrm{kg}$ do BHT e do TBHQ provocou o aumento ainda maior da estabilidade oxidativa do sebo bovino.

Ressalte-se que um incremento de algumas horas no período de indução de um produto triglicerídico pode corresponder a várias semanas adicionais de tempo de armazenagem sem rancificação considerável do material considerado (ANTONIASSI, 2001). Tal fato sugere que o BHT e o IONOL seriam bons antioxidantes para este insumo tão relevante na cadeia produtiva nacional de biodiesel. No entanto, estudos ainda devem ser conduzidos de modo a otimizar essa dosagem, considerando particularmente aspectos técnicos (e.g., caraterísticas do sebo, estabilidade oxidativa, processo produtivo), econômicos e ambientais.

\section{CONCLUSÕES}

O sebo utilizado é uma gordura animal com características típicas da sua origem bovina, inclusive com baixo teor de insaturados. A adição dos antioxidantes BHT e IONOL eleva consideravelmente a estabilidade oxidativa do sebo bovino, podendo aumentar até quatro vezes o valor do período de indução deste insumo da cadeia produtiva do biodiesel. O IONOL se mostrou um bom antioxidante no aumento da estabilidade do sebo bovino, superando o BHT na dosagem de $5000 \mathrm{mg} / \mathrm{kg}$. Também, na faixa de dosagem estudada (1000 mg/kg e $5000 \mathrm{mg} / \mathrm{kg}$ ), o aumento na concentração de antioxidante ainda eleva o período de indução do material sebáceo estudado.

\section{REFERÊNCIAS BIBLIOGRÁFICAS}

ALVES, I. A. et al. Avaliação do Uso de Antioxidantes Comerciais na Estabilidade Oxidativa de Sebo Bovino. X CONNEPI - Congresso
Norte Nordeste de Pesquisa e Inovação. Anais...Rio Branco: 2015

AMERICAN OIL CHEMISTS' SOCIETY. Official methods and recommended practices of the American Oil Chemists' Society. AOCS Official method Cd 8-53. 4th. ed. Champaign, USA: [s.n.].

AMERICAN OIL CHEMISTS' SOCIETY. Official Methods and Recommended Practices of the AOCS. 6th editio ed. [s.l: s.n.].

ANTONIASSI, R. Métodos de avaliação da estabilidade oxidativa de óleos e gorduras. Boletim Centro de Pesquisa e Processamento de Alimentos (CEPPA), v. 19, n. 2, p. 353-380, 2001.

ARAÚJO, S. V. et al. FTIR assessment of the oxidation process of castor oil FAME submitted to PetroOXY and Rancimat methods. Fuel Processing Technology, v. 92, n. 5, p. 1152-1155, 2011.

ASSOCIAÇÃO BRASILEIRA DE NORMAS TÉCNICAS. ABNT NBR 13573, Amostragem de insumos químicos para curtimento e acabamento de couros. [s.1.] ABNT, 2012.

BRASIL. Biodiesel. O novo combustível do Brasil. Programa Nacional de Produção e Uso do Biodiesel. Ministério de Minas e Energia. Disponível em: <www.mme.gov.br>. Acesso em: 13 jan. 2016.

BRASIL. Boletim mensal dos combustíveis renováveis. Ministério de Minas e Energia. Disponível em: <www.mme.gov.br>. Acesso em: 8 ago. 2016a.

BRASIL. Boletim mensal do biodiesel. Agência Nacional do Petróleo, Gás Natural e Biocombustíveis. Disponível em: 〈www.anp.gov.br〉. Acesso em: 27 jul. 2016 b.

BSI BRITISH STANDARDS. Fat and oil derivatives. Fatty acid methyl esters (FAME). Determination of oxidation stability (accelerated oxidation test). [s.1.] BSI, 2003.

CASTANHEIRA, É. G. et al. Environmental sustainability of biodiesel in Brazil. Energy Policy, v. 65, p. 680-691, 2014.

FARIAS, P. N. et al. Estudo da estabilidade termoxidativa de biodiesel e ecodieseis de sebo bovino. Anais do VIII Salão de Iniciação Científica/I Encontro de Pesquisa e Pós-Graduação da Universidade do Estado do Rio Grande do Norte. Anais...2012

MARTINS, R. et al. O Biodiesel de Sebo Bovino no Brasil. Informações Econômicas, v. 41, n. 5, p. 15, 
2011.

MORETTO, E.; FETT, R. Tecnologia de óleos e gorduras vegetais na indústria de alimentos. São Paulo: Varela Editora e Livraria Ltda, 1998.

ORIVES, J. R. et al. Multiresponse optimisation on biodiesel obtained through a ternary mixture of vegetable oil and animal fat: Simplex-centroid mixture design application. Energy Conversion and Management, v. 79, p. 398-404, 2014.

PINTO, V. DE L. Efeitos do Envelhecimento na Degradação Termoxidativa do Sebo Bovino e seus Biocombustíveis via Análise de UV-VIS. [s.l.] Universidade do Estado do Rio Grande do Norte, 2014.

RINCÓN, L. E.; JARAMILLO, J. J.; CARDONA, C. A. Comparison of feedstocks and technologies for biodiesel production: An environmental and technoeconomic evaluation. Renewable Energy, v. 69, p. 479-487, 2014.

SANTOS, F. F. P. DOS. Avaliação de antioxidantes aplicados à produção de biodiesel. [s.1.] Universidade Federal do Ceará, 2013.

SOUZA, F. H. N. et al. UV-Visible Spectroscopy Study of Oxidative Degradation of Sunflower Biodiesel. Energy Science and Technology, v. 2, n. 2, p. 56-61, 2011.

\section{AGRADECIMENTOS}

Os autores agradecem ao Conselho Nacional de Desenvolvimento Científico e Tecnológico (CNPq) pelo suporte financeiro e pelas bolsas ITI-A concedidas por meio da Chamada CNPqSETEC/MEC $\mathrm{n}^{\circ} 17 / 2014 \quad-\quad$ Linha 1 (Processo n 468875/2014-0), à Coordenação de Aperfeiçoamento de Pessoal de Nível Superior (CAPES) pela bolsa de mestrado concedida, ao Programa Institucional de Bolsas do IFCE/CNPq pelas bolsas PIBICJR concedidas, à Pró-Reitoria de Pesquisa, Pós-Graduação e Inovação do Instituto Federal de Educação, Ciência e Tecnologia do Ceará IFCE pelo suporte financeiro obtido por meio do Edital $N^{\circ} 04 / 2015$ - PRPI - PROINFRA/IFCE - Linha 4. Também agradecem aos técnicos, bolsistas e estagiários do Laboratório de Processos e Análises Químicas do IFCE - Campus Fortaleza e do Núcleo de Pesquisas em Lubrificantes Prof. Ícaro de Souza Moreira da Universidade Federal do Ceará pela colaboração em alguns dos ensaios realizados. 